\title{
Symbol by Symbol Doppler Rate Estimation for Highly Mobile Underwater OFDM
}

\author{
Nathan Parrish, Sumit Roy \\ Department of Electrical Engineering \\ University of Washington \\ Seattle, Washington 98195 \\ Email: nparrish,sroy@u.washington.edu
}

\author{
Payman Arabshahi \\ Applied Physics Laboratory \\ University of Washington \\ Seattle, Washington 98105 \\ Email: payman@apl.washington.edu
}

\begin{abstract}
We propose an OFDM receiver capable of estimating and correcting, on a symbol-by-symbol basis, the subcarrier dependent Doppler shifting due to the movement of source and receiver in an underwater acoustic network. We propose two methods of estimation: one of which is based upon the marginal maximum likelihood principle, and one of which is ad-hoc. We compare the performance of both estimators to the Cramer-Rao lower bound. We show through simulation that the proposed receiver design performs well for a source that is accelerating at $0.29 \mathrm{~m} / \mathrm{s}^{2}$.
\end{abstract}

\section{INTRODUCTION}

The wideband modulation scheme Orthogonal Frequency Division Multiplexing (OFDM) has recently generated much interest in the context of underwater acoustic communications for its potential to increase achievable data rates. OFDM through the use of a cyclic prefix (CP) or guard interval - transforms the frequency selective underwater channel into multiple frequency non-selective orthogonal channels. Additionally, OFDM is easily implemented via the Fast Fourier Transform (FFT). However, because of the relatively low propagation speed of acoustic sound in water, the motion of the source and receiver causes non-uniform Doppler shifting among the subcarriers at different frequencies. If not compensated, this Doppler shift destroys the orthogonality of the subcarriers causing severe inter-carrier interference (ICI) at the receiver. For networks of highly mobile underwater vehicles, such as the Remus line of AUVs [1] which can travel at speeds of up to $2.6 \mathrm{~m} / \mathrm{s}$, receiver algorithms must be able to correct for this Doppler shift.

For underwater channels in which the Doppler shift on all component multipaths is approximately equal, it has been shown that the resulting (non-uniform) Doppler among subcarriers described above can be estimated via a single channel parameter - the Doppler rate (vehicle speed divided by the speed of sound in the medium). Li et al. [2] recently showed that by re-sampling the received signal at a period proportional to the Doppler rate, the frequency dependent Doppler shift can be eliminated. Therefore, conventional pilot assisted estimation methods for joint channel and carrier frequency offset estimation can be used to recover the transmitted data symbols.

Doppler rate estimation has been accomplished in [2] and [3] via a preamble/postamble structure that assumes that this rate is constant over a block of OFDM symbols. Sharif showed in [4] that the Doppler rate can be estimated by a pair of LFM chirps, and Li extended this method for use in OFDM. In [3], the Doppler rate is estimated over a block of OFDM symbols via a preamble that contains a repeated OFDM symbol with a cyclic prefix. Both of the above designs estimate the Doppler rate independent of the OFDM symbols and assume that the rate remains constant. This poses limitations for a network of highly mobile underwater nodes. First, if the underwater nodes change direction and/or speed often, then the Doppler rate must be re-estimated often which requires re-transmission of the pre-amble and a consequent loss in data rate. Furthermore, if the Doppler rate changes significantly over the course of an OFDM block, then reception will fail due to increased ICI.

In this paper, we propose a receiver structure that alleviates these problems by estimating (and correcting for) the Doppler rate on a symbol-by-symbol basis. A block diagram of our proposed receiver structure is shown in Figure (1). The transmitted block of OFDM symbols is preceded by a preamble that allows for initial timing and Doppler rate estimation. Joint carrier frequency offset and channel estimation are then performed on the first symbol assuming that the the Doppler rate estimated during the preamble is equal to the actual Doppler rate during the first OFDM symbol. The recovered data is used to update the estimate of the Doppler rate which is then used for decoding of the next OFDM symbol; this process is continued on a symbol-by-symbol basis. We develop two algorithms for the Doppler rate update: one ad-hoc, and the other based on the Marginal Maximum Likelihood principle. We show through simulation that such a receiver design can support increased data rates with reduced probability of symbol error when the relative velocity between the transmitter and receiver changes over a velocity range commensurate with the Remus 6000 AUV.

Notation: Superscript $(.)^{(i)}$ represents the $i$-th OFDM symbol vector. Lowercase bold face letters indicate column vectors, and uppercase bold face letters indicate matrices. The superscript $(.)^{T}$ indicates transpose and $(.)^{H}$ indicates conjugate transpose. E[.] indicates the expected value. Finally $\hat{.}$ indicates a value acquired via estimation. 


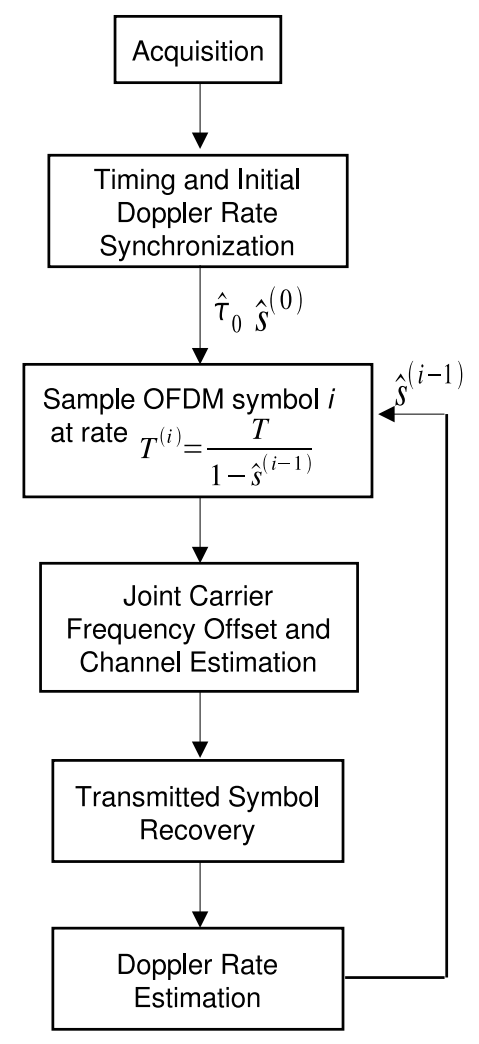

Fig. 1. Receiver Block Diagram

\section{OFDM SignAl AND CHANNEL Model}

A baseband representation of CP-OFDM for a transmit pulse shape $g(t)$ is given by

$$
x(t)=\sum_{i=0}^{\infty} \frac{1}{\sqrt{K}} \sum_{k=-\frac{K}{2}}^{\frac{K}{2}-1} d_{k}^{(i)} e^{j 2 \pi \frac{k}{T K}\left(t-T_{g}-i T_{s}\right)} g\left(t-i T_{s}\right)
$$

where $d_{k}^{(i)}$ represents the symbol on the $k$-th sub-carrier within the $i$-th OFDM symbol. $K$ is the total number of subcarriers and $T_{s}$ is the OFDM symbol period, given by $T_{s}=T\left(N_{g}+\right.$ $K$ ), where $N_{g}$ is the number of symbols in the cyclic prefix and $T=1 / B$ is the sampling period for a channel bandwidth of $B$. The separation between OFDM subcarriers is $\Delta f=$ $\frac{1}{T K}$.

The baseband symbol is modulated at carrier frequency $f_{c}$ to yield the corresponding transmitted passband signal $f(t)=$ $\operatorname{Re}\left\{x(t) e^{j 2 \pi f_{c} t}\right\}$ which is transmitted over an acoustic channel with time varying channel impulse response

$$
h(\tau, t)=\sum_{l=0}^{L-1} \alpha_{l}(t) \delta\left(t-\tau_{l}(t)\right)
$$

where $\tau_{l}(t)$ and $\alpha_{l}(t)$ are the delay and complex gain of path $l$ at time $t$. In (2), there exist $L$ discrete multipath components.
The received signal $r(t)$ plus additive white Gaussian noise, $w(t)$, is

$$
r(t)=\operatorname{Re}\left\{\sum_{l=0}^{L-1} \alpha_{l}(t) x\left(t-\tau_{l}(t)\right) e^{j 2 \pi f_{c}\left(t-\tau_{l}(t)\right)}+w(t)\right\}
$$

which is downconverted to give

$$
y(t)=\sum_{l=0}^{L-1} \alpha_{l}(t) x\left(t-\tau_{l}(t)\right) e^{-j 2 \pi\left(f_{c} \tau_{l}(t)+\Delta f_{c} t\right)}+w(t)
$$

In (4), $\Delta f_{c}$ is the carrier frequency offset between the source and receiver. We assume that the time variation in the delay is due primarily to the motion of the source/receiver, and is approximately equal on all paths. Therefore, for relative velocity $v(t)$ between the source-receiver pair, $\tau_{l}(t)=\tau_{l}+\int_{0}^{t} s(t) d t$ where $s(t)=\frac{v(t)}{c}$, where the first term represents the delay at a reference time $t=0$.

Substituting for $\tau_{l}(t)$ and $x(t)$ into equation (4), the received signal (neglecting the noise term) can be expressed as

$$
\begin{aligned}
y(t)= & \sum_{i=0}^{\infty} e^{-j 2 \pi\left(f_{c} \int_{0}^{t} s(t) d t+\Delta f_{c} t\right)} \frac{1}{\sqrt{K}} \\
& \sum_{k=-K / 2}^{K / 2-1} d_{k}^{(i)} e^{j 2 \pi \frac{k}{T K}\left(t-T_{g}-i T_{s}-\int_{0}^{t} s(t) d t\right)} H\left(f_{k}, t\right)
\end{aligned}
$$

where $H\left(f_{k}, t\right)$ is the channel transfer function at time $t$ on the $k$ th subcarrier.

$$
\begin{gathered}
H\left(f_{k}, t\right)=\sum_{l=0}^{L-1} b_{l}(t) e^{-j 2 \pi \frac{k \tau_{l}}{T K}} \\
b_{l}(t)=\alpha_{l}(t) g\left(t-i T_{s}-\tau_{l}-\int_{0}^{t} s(t) d t\right) e^{-j 2 \pi f_{c} \tau_{l}}
\end{gathered}
$$

We denote the time when the leading edge of the $i$ th symbol arrives at the receiver as $t^{(i)}$. We also assume that $s(t)$ and $\alpha_{l}(t)$ are slowly time varying with respect to the OFDM symbol interval, therefore over the interval $t=\left[t^{(i)}, t^{(i+1)}\right]$

$$
\begin{aligned}
& s(t)=s^{(i)} \\
& a_{l}(t)=a_{l}^{(i)}
\end{aligned}
$$

The propagation of symbols from source to receiver is shown in figure (2). In this figure we also indicate the delay spread of the channel, denoting the time that the trailing edge of the $i$ th symbol arrives at the receiver as $t^{\prime}(i+1)$. From equation (7), we can see that symbol 0 , transmitted over interval $\left[0, T_{s}\right]$ arrives at the receiver over interval $\left[t^{(0)}, t^{\prime(1)}\right]=$ $\left[\frac{\tau_{\min }}{1-s^{(0)}}, \frac{T_{s}+\tau_{\max }}{1-s^{(0)}}\right]$. It can also be shown that:

$$
\begin{aligned}
& t^{(i)}=t^{(i-1)}+\frac{T_{s}}{1-s^{(i)}} \\
& t^{\prime(i)}=t^{\prime(i-1)}+\frac{T_{s}}{1-s^{(i)}}
\end{aligned}
$$

Additionally, we assume that $N_{g} T>\tau_{\max }-\tau_{\min }$, ie. that all the ISI is contained within the cyclic prefix (as indicated 


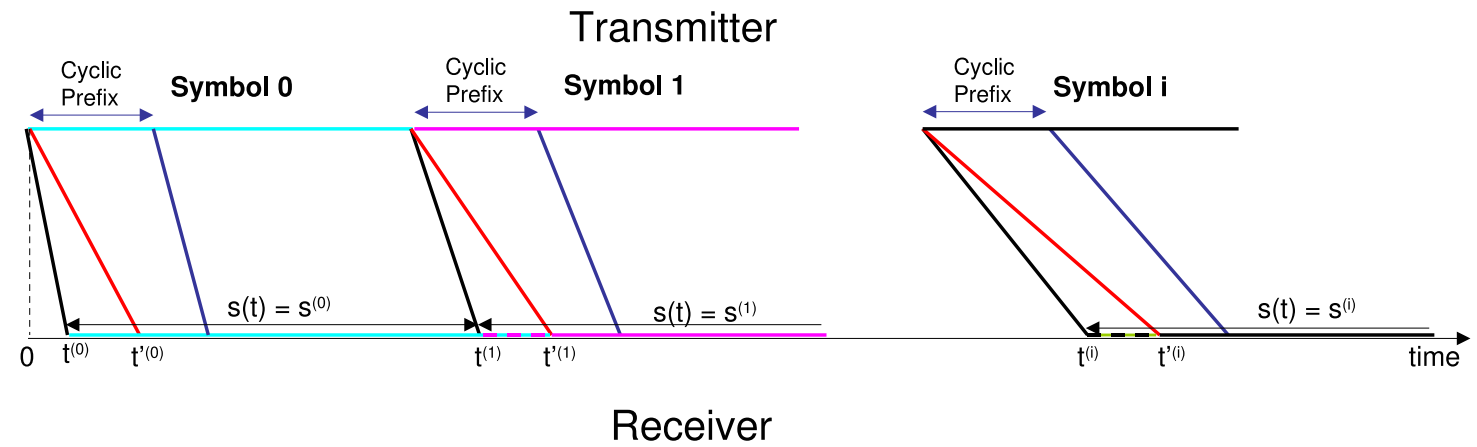

Fig. 2. Propagation of OFDM Symbols from Transmitter to Receiver under the assumption that the Doppler Rate is slowly time varying. Black lines (red lines) from source to receiver represent the propagation on the path with the minimum (maximum) delay. Notice that all ISI from symbol $i-1$ arrives during the cyclic prefix of symbol $i$ arrive within the Cyclic Prefix.

in figure (2)). Since the cyclic prefix will be discarded by the OFDM receiver, we disregard the ISI and express the received signal over the interval $\left[t^{(i)}, t^{(i+1)}\right]$ as:

$$
\begin{aligned}
y^{(i)}\left(t^{\prime}\right)= & y\left(t^{(i)}+t^{\prime}\right) \\
= & e^{-j 2 \pi\left(f_{c} s^{(i)}+\Delta f_{c}\right) t^{\prime}} \frac{1}{\sqrt{K}} \cdot \\
& \sum_{k=-K / 2}^{K / 2-1} d_{k}^{(i)} e^{j 2 \pi \frac{k}{T K}\left(\left(t^{\prime}\right)\left(1-s^{(i)}\right)-T_{g}\right)} H^{(i)}\left(f_{k}\right)
\end{aligned}
$$

where $H^{(i)}\left(f_{k}\right)$ is:

$$
\begin{gathered}
H^{(i)}\left(f_{k}\right)=\sum_{l=0}^{L-1} b_{l}^{(i)} e^{-j 2 \pi \frac{k\left(\tau_{l}-\tau_{\min }\right)}{T K}} \\
b_{l}^{(i)}=\alpha_{l}^{(i)} g\left(t^{\prime}-\left(\tau_{l}-\tau_{\min }\right)\right) e^{-j 2 \pi\left(f_{c}\left(\tau_{l}+t^{(i)}\right)+\Delta f_{c} t^{(i)}\right)}
\end{gathered}
$$

We note the three effects of the Doppler Rate in (9) which were also cited in [2]. The first is that the $i$-th received OFDM symbol which was transmitted over a time period of $T_{s}$ seconds is received over a time period of $\frac{T_{s}}{1-s^{(i)}}+\tau_{\max }-\tau_{\min }$ seconds. This indicates a spreading of the received signal due to the multipath of the channel as well as a 'dilation' due to the Doppler rate. The second effect is the subcarrier dependent Doppler shift $\Delta f_{k}=-s^{(i)} k / K T$. Finally, there is an effective carrier frequency offset of $f_{c} s^{(i)}+\Delta f_{c}$ across all subcarriers.

\section{DisCRETE TIME MODEL}

We develop an equivalent discrete time model by sampling Eq. (9). In order to completely eliminate the subcarrier dependent Doppler shift from the received signal samples, the $i$ th OFDM symbol must be sampled at rate $T^{(i)}=\frac{T}{1-s^{(i)}}$. However, since we do not a-priori know $s^{(i)}$, we sample at rate $T^{(i)}=\frac{T}{1-s^{(i-1)}}$ (we show later how the estimate of $s$ is generated from OFDM symbol $i-1$ ). By sampling symbol $i$ at this rate, the received discrete time samples are given as:

$y_{n}^{(i)}=e^{-j 2 \pi \epsilon^{(i)} n T} \sum_{k=-K / 2}^{K / 2-1} d_{k}^{(i)} e^{j 2 \pi \frac{k}{K}\left(n \beta^{(i)}-N_{g}\right)} H^{(i)}\left(f_{k}\right)+w_{n}^{(i)}$ where $\epsilon^{(i)}=\frac{f_{c} s^{(i)}}{1-s^{(i-1)}}+\frac{\Delta f_{c}}{1-s^{(i-1)}}$ and $\beta^{(i)}=\frac{1-s^{(i)}}{1-s^{(i-1)}}$. The final term in (12) is a zero-mean, circularly symmetric complex Gaussian noise with variance $\sigma_{w}^{2}$ that is i.i.d in the index $n$.

After the first $N_{g}$ samples (which form the cyclic prefix) are removed, the remaining $K$ samples in Equation (12) are conveniently expressed in vector-matrix form as

$$
\mathbf{y}^{(i)}=\mathbf{M}\left(\epsilon^{(i)}\right) \mathbf{W}_{\beta^{(i)}}^{H} \mathbf{D}^{(i)} \mathbf{h}^{(i)}+\mathbf{w}^{(i)}
$$

where $\mathbf{y}^{(i)}$ is the vector of the $K$ discrete time samples, $\mathbf{M}\left(\epsilon^{(i)}\right)=\operatorname{diag}\left[1, e^{-j 2 \pi \epsilon^{(i)} T}, \ldots, e^{-j 2 \pi \epsilon^{(i)}(K-1) T}\right]$ is the effective carrier frequency offset, $\mathbf{h}=$ $\left[H^{(i)}\left(f_{-K / 2}\right), . ., H^{(i)}\left(f_{0}\right), \ldots, H^{(i)}\left(f_{K / 2-1}\right)\right]^{T} \quad$ is the channel transfer function, $\mathbf{W}_{\beta^{(i)}}$ is a matrix with entry $\left[W_{\beta^{(i)}}\right]_{k, n}=\frac{1}{\sqrt{K}} e^{-j 2 \pi \frac{k\left((n+N g) \beta^{(i)}-N_{g}\right)}{K}}, \mathbf{D}^{(i)}$ is a diagonal matrix of the data symbols, and $\mathbf{w}^{(i)}$ is a zero-mean iid Gaussian noise vector with covariance matrix $\sigma_{w}^{2} \mathbf{I}$.

Assuming that the effective carrier frequency offset is known, we form

$$
\begin{aligned}
\tilde{\mathbf{y}}^{(i)} & =\mathbf{M}^{H}\left(\epsilon^{(i)}\right) \mathbf{y}^{(i)} \\
& =\mathbf{W}_{\beta^{(i)}}^{H} \mathbf{D}^{(i)} \mathbf{h}^{(i)}+\mathbf{M}^{H}\left(\epsilon^{(i)}\right) \mathbf{w}^{(i)}
\end{aligned}
$$

Taking the DFT of the received data, we get

$$
\begin{aligned}
\mathbf{W}_{\mathbf{1}} \tilde{\mathbf{y}}^{(i)} & =\mathbf{W}_{1} \mathbf{W}_{\beta^{(i)}}^{H} \mathbf{D}^{(i)} \mathbf{h}^{(i)}+\mathbf{q}^{(i)} \\
\mathbf{q}^{(i)} & =\mathbf{W}_{1}\left(\mathbf{M}^{H}\left(\epsilon^{(i)}\right)\right) \mathbf{w}^{(i)}
\end{aligned}
$$

It is noted that the vector $\mathbf{q}^{(i)}$ is a unitary transform of the vector $\mathbf{w}^{(i)}$ and therefore, has the same statistics. We denote the covariance matrix of $\mathbf{q}^{(i)}$ as $\sigma_{q}^{2} \mathbf{I}$. From (15) the $m$ th received subcarrier symbol on the $i$ th received OFDM symbol, for $m=[-K / 2, \ldots, K / 2-1]$ is:

$$
\begin{aligned}
z_{m}^{(i)=} & \frac{1}{K} \sum_{k=-K / 2}^{K / 2-1} H^{(i)}\left(f_{k}\right) d_{k}^{(i)} e^{\left(j 2 \pi \frac{k}{K} N_{g}\left(\beta^{(i)}-1\right)\right)} . \\
& \sum_{n=0}^{K-1} e^{j 2 \pi \frac{n}{K}\left(k \beta^{(i)}-m\right)}+q_{m}^{(i)}
\end{aligned}
$$




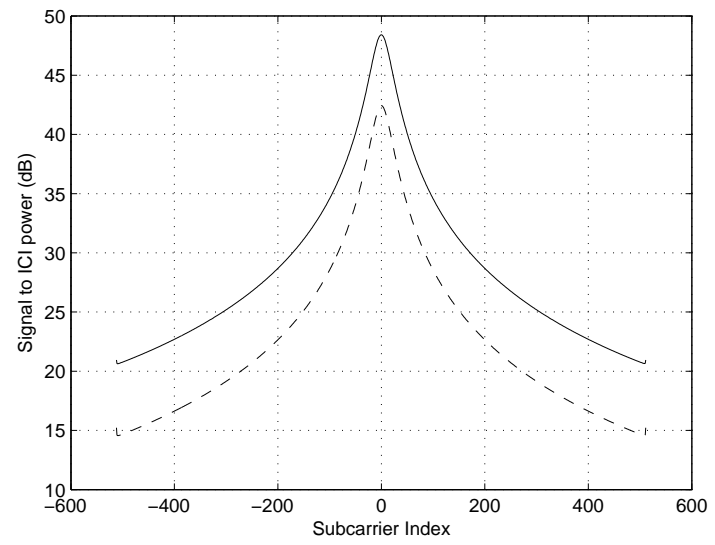

Fig. 3. $\mathrm{E}\left[\frac{\left|\mathrm{Sig}_{m}\right|^{2}}{\left|\mathrm{ICI}_{m}\right|^{2}}\right]$, ratio of signal power to ICI power per subcarrier $K=1024$ subcarriers, $\Delta f=2.8 \mathrm{~Hz}$, and $\Delta v^{(i)}=0.156 \mathrm{~m} / \mathrm{s}$ (solid line) $\Delta v^{(i)}=0.302 \mathrm{~m} / \mathrm{s}$ (dashed line).

We define the matrix $\mathbf{G}(\beta)$ with elements

$$
\begin{gathered}
{\left[\mathbf{G}\left(\beta^{(i)}\right)\right]_{m, k}=\frac{1}{K} \sum_{n=0}^{K-1} e^{j 2 \pi \frac{n}{K}\left(k\left(\beta^{(i)}\right)-m\right)}} \\
{\left[\mathbf{G}\left(\beta^{(i)}\right)\right]_{m, k}=\frac{1}{K} e^{\frac{j \pi\left(k\left(\beta^{(i)}\right)-m\right)(K-1)}{K}} \frac{\sin \left(\pi\left(k\left(\beta^{(i)}\right)-m\right)\right)}{\sin \left(\frac{\pi\left(k\left(\beta^{(i)}\right)-m\right)}{K}\right)}}
\end{gathered}
$$

and the matrix $\mathbf{N}_{G}\left(\beta^{(i)}\right)$ as a diagonol matrix with the $(k, k)$ element given as $e^{j 2 \pi \frac{k}{K}\left(N_{g}\left(\beta^{(i)}-1\right)\right)}$. Finally, we can write the received signal vector as:

$$
\mathbf{z}=\mathbf{G}\left(\beta^{(i)}\right) \mathbf{N}_{G}\left(\beta^{(i)}\right) \mathbf{D}^{(i)} \mathbf{h}^{(i)}+\mathbf{q}^{(i)}
$$

The matrix $\mathbf{G}\left(\beta^{(i)}\right)$, therefore, creates an ICI pattern, where the ICI on the $m$ th subcarrier is:

$$
\mathrm{ICI}_{m}=\sum_{k \neq m} H^{(i)}\left(f_{k}\right) d_{k}^{(i)}\left[\mathbf{G}\left(\beta^{(i)}\right) \mathbf{N}_{G}\left(\beta^{(i)}\right)\right]_{(m, k)}
$$

and the useful signal is:

$$
\operatorname{Sig}_{m}=H^{(i)}\left(f_{m}\right) d_{m}^{(i)}\left[\mathbf{G}\left(\beta^{(i)}\right) \mathbf{N}_{G}\left(\beta^{(i)}\right)\right]_{(m, m)}
$$

Figure (3) plots the ratio of signal power to ICI power, $\mathrm{E}\left[\frac{\left|\mathrm{Sig}_{m}\right|^{2}}{\left|\mathrm{ICI}_{m}\right|^{2}}\right]$, for two different values of $\beta^{(i)}$, assuming that $\mathrm{E}\left[\left|H^{(i)}\left(f_{k}\right)\right|^{2}\right]=\mathrm{E}\left[\left|d_{k}^{(i)}\right|^{2}\right]=1$. However, rather than considering $\beta^{(i)}$, it is more instructional to consider the relative acceleration of the transmitter/receiver directly. Therefore, the figure indicates the $\Delta v^{(i)}$, which is the change in velocity from one OFDM symbol to the next.

\section{RECEIVER DESIGN}

The receiver schematic is shown in Figure (4). The incoming symbol is sampled at rate $T^{(i)}=\frac{T}{1-\hat{s}^{(i-1)}}$, where $\hat{s}^{(i-1)}$ is the estimate of the Doppler Rate for the previous symbol or from initial synchronization. Our receiver operates in two stages. The first stage involves transmitted symbol recovery via joint carrier frequency offset and channel estimation under the

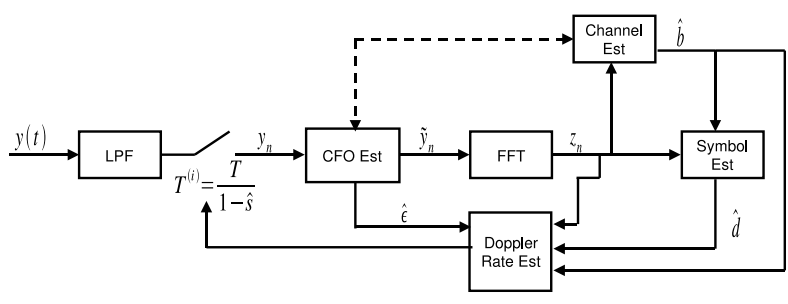

Fig. 4. Receiver Design

assumption that $\beta^{(i)}=1$. The second stage uses the carrier frequency offset estimate, transmitted symbol estimates, and the channel estimates found in the first stage to estimate the Doppler rate. The next symbol is sampled then at rate $T^{(i+1)}=\frac{T}{1-\hat{s}^{(i)}}$ given by the updated Doppler rate.

In order to perform joint channel and carrier frequency offset estimation, we first assume that the channel is a tapped delay line with $N_{g}$ taps spaced at $T^{(i)}$ intervals. By further assuming $\beta=1$, the model for the received samples (13) becomes (we drop the superscript $(i)$ for simplicity)

$$
\mathbf{y}=\mathbf{M}(\epsilon) \mathbf{W}_{1}^{H} \mathbf{D V b}+\mathbf{w}
$$

where $\mathbf{V}$ is a $K$ by $N_{g}$ matrix equal to the first $K$ columns of $\mathbf{W}_{1}$ and $\mathbf{b}$ is the vector of tap delay line channel coefficients. A subset of the transmitted symbols are known pilot symbols, whose location is given by the matrix $\mathbf{S}$. If there are $p$ pilot symbols, then $\mathbf{S}$ is a $p$ by $K$ matrix with row $p$ having a 1 in the column corresponding to the $p$ th pilot location and zeros elsewhere.

We perform joint channel and carrier frequency offset estimation by the method proposed in [5]. We first assume a range of values for $\epsilon$. For each candidate, which we denote as $\tilde{\epsilon}$, we can form a least squares estimate of the channel impulse response as:

$$
\hat{\mathbf{b}}=(\mathbf{S D V})^{\dagger} \mathbf{S} \mathbf{W}_{1} \mathbf{M}^{H}(\tilde{\epsilon}) \mathbf{y}
$$

where $(.)^{\dagger}$ indicates the pseudoinverse. From this estimate of the channel impulse response, we can form the estimate of the channel transfer function as

$$
\hat{\mathbf{h}}=\mathbf{V} \hat{\mathbf{b}}
$$

Finally, we recover the transmitted data symbols via

$$
\tilde{d}_{k}=\frac{z_{k}}{\hat{h}_{k}}
$$

For each candidate $\tilde{\epsilon}$, the mean square error (MSE) between the known pilot symbols and the $\tilde{d}_{k}$ is computed. The $\tilde{\epsilon}$ which gives the lowest MSE is chosen as $\hat{\epsilon}$.

The data symbols in an underwater communications system are typically channel coded, so that the soft estimates given by (26) are forwarded down the receiver chain for de-interleaving and decoding before a final symbol decision is reached. However, we also immediately form a hard decision of the transmitted data symbols by simply decoding the soft estimates to the nearest neighbor in the transmitted symbol constellation, and we denote these estimates as $\hat{d}_{k}$. 


\section{Doppler Rate Estimation}

\section{A. Initial Estimation}

In [6] it was shown that the joint maximum likelihood estimate of the Doppler rate and delay $\hat{s}$ and $\hat{\tau}_{0}$ for a nondispersive channel (ie, a channel for which $L=1$ ) can be computed by transmitting a known training signal and using a bank of sliding correlators at the receiver. Each correlator is tuned to a different Doppler rate, and the maximum likelihood estimate is a 2-D grid search over time (for $\hat{\tau}_{0}$ ) and the correlators (for $\hat{s}$ ). In [3], the estimation is more effectively carried out for dispersive, underwater channel by transmitting a repeated OFDM symbol with a Cyclic Prefix, and using a sliding bank of self-correlators, each tuned to a different Doppler rate. In [3] the Doppler rate was assumed constant over packet of several OFDM symbols. We adopt this method of initial Doppler rate and delay estimation; however, we add a method for tracking the change in Doppler rate from OFDM symbol to symbol.

\section{B. Marginal Maximum Likelihood Estimator}

We wish to develop a maximum likelihood estimator for $\beta$ from (20). In order to form (20), we use $\hat{\epsilon}$ given by the joint channel and carrier frequency offset estimation. We can represent $\mathbf{F}(\tilde{\beta})=\mathbf{G}(\tilde{\beta}) \mathbf{N}_{G}(\tilde{\beta}) \mathbf{D V}$, where we form $\mathbf{D}$ from a combination of known pilot symbols and our estimates given in section (IV). Since the noise is assumed iid, zero mean Gaussian, the maximum likelihood estimate of $(\beta, \mathbf{b})$ is achieved by maximizing the probability:

$p(\mathbf{z} \mid \tilde{\beta}, \tilde{\mathbf{b}})=\frac{1}{\left(\pi \sigma_{q}^{2}\right)^{K}} \exp \left\{-\frac{1}{\sigma_{q}^{2}}(\mathbf{z}-\mathbf{F}(\tilde{\beta}) \tilde{\mathbf{b}})^{H}(\mathbf{z}-\mathbf{F}(\tilde{\beta}) \tilde{\mathbf{b}})\right\}$

over all possible pairs $\tilde{\beta}, \tilde{\mathbf{b}}$. This is achieved by maximizing:

$$
\begin{aligned}
\Lambda(z \mid \tilde{\beta}, \tilde{\mathbf{b}})= & \exp \left\{-\frac{1}{\sigma_{q}^{2}}\left(|\mathbf{z}|^{2}-\mathbf{z}^{H} \mathbf{F}(\tilde{\beta}) \tilde{\mathbf{b}}-\tilde{\mathbf{b}}^{H} \mathbf{F}(\tilde{\beta})^{H} \mathbf{z}\right.\right. \\
& \left.\left.+\tilde{\mathbf{b}}^{H} \mathbf{F}(\tilde{\beta})^{H} \mathbf{F}(\tilde{\beta}) \tilde{\mathbf{b}}\right)\right\}
\end{aligned}
$$

The first term in (28) has no impact on the maximization. Furthermore, in order to estimate $\beta$ independently of $\mathbf{b}$, we develop a marginal likelihood estimator, as shown in [7], by averaging over the probability distribution of $\tilde{\mathbf{b}}$. We do this by performing the operation $\int_{-\infty}^{\infty} \Lambda(z \mid \tilde{\beta}, \tilde{\mathbf{b}}) \mathrm{p}(\tilde{\mathbf{b}}) d \tilde{\mathbf{b}}$. We substitute (28) into this equation. Assuming that the elements of the channel impulse response are independent Rayleigh fading arrivals, the marginal maximum likelihood estimate of $\beta$ is given by:

$$
\begin{aligned}
\Lambda(z \mid \tilde{\beta})= & \frac{1}{\operatorname{det}\left[\mathbf{I}+\frac{1}{\sigma^{2}} \mathbf{R}_{\tilde{\mathbf{b}}} \mathbf{F}(\tilde{\beta})^{H} \mathbf{F}(\tilde{\beta})\right]} \\
& \exp \left\{\left(\frac{1}{\sigma^{2}}\right)^{2} \mathbf{z}^{H} \mathbf{F}(\tilde{\beta}) \cdot\right. \\
& {\left.\left.\left[\mathbf{R}_{\tilde{\mathbf{b}}}^{-1}+\frac{1}{\sigma^{2}} \mathbf{F}(\tilde{\beta})^{H} \mathbf{F}(\tilde{\beta})\right]^{-1} \mathbf{F}(\tilde{\beta})^{H} \mathbf{z}\right]\right\}(29) }
\end{aligned}
$$

At high SNRs, it can be shown that the maximum of (29) can be found by maximizing:

$$
\Lambda(z \mid \tilde{\beta})=\mathbf{z}^{H} \mathbf{F}(\tilde{\beta})\left[\mathbf{F}(\tilde{\beta})^{H} \mathbf{F}(\tilde{\beta})\right]^{-1} \mathbf{F}(\tilde{\beta})^{H} \mathbf{z}
$$

We denote the value of $\beta$ which maximizes (30) as $\hat{\beta}_{\mathrm{MML}}$.

\section{An Ad-hoc Estimator}

The marginal maximum likelihood estimator presented in the preceding section requires a grid search. In this section, we develop an ad-hoc estimator of $\beta$, which does not require a grid search, but which performs sub-optimally in comparison to the MML estimator.

From section III, it is shown that the received sample on the $k$ th subcarrier can be expressed as:

$$
z_{k}=c_{k} e^{\frac{j \pi k(\beta-1)}{K}\left(K-1+2 N_{g}\right)} d_{k} h_{k}+q_{k}+\mathrm{ICI}_{k}
$$

The first term in (31) is comprised of $c_{k}$, a real attenuation factor given by the $\sin$ terms in element $(k, k)$ of (19), the phase offset due to $\left[\mathbf{G}(\beta) \mathbf{N}_{g}(\beta)\right]_{(k, k)}$, the transmitted data bit, and the channel transfer function. The other terms are the AWGN and $\mathrm{ICI}_{k}$ as defined in equation (21). If we assume that the the ICI is additional zero-mean AWGN, then we can estimate the angle in the above equation as:

$$
\frac{\pi k(\beta-1)}{K}\left(K-1+2 N_{g}\right)=\angle\left(d_{k}^{*}\left(\frac{z_{k}}{h_{k}}\right)\right)
$$

and

$$
\hat{\beta}_{k}=\frac{\angle\left(d_{k}^{*}\left(\frac{z_{k}}{h_{k}}\right)\right) K}{\left(K-1+2 N_{g}\right) \pi k}+1
$$

Therefore, $\hat{\beta}$ can be found by averaging the $\hat{\beta}_{k}$.

$$
\hat{\beta}=\frac{1}{K} \sum \hat{\beta}_{k}
$$

Note that this estimate of $\beta$ is similar to that used by Stojanovic in [8].

If we assume that the ICI term is additional Gaussian noise, then $\hat{\beta}$ is an unbiased estimator of $\beta$ as follows.

$$
\begin{aligned}
\mathrm{E}\left[\angle\left(d_{k}^{*}\left(\frac{z_{k}}{h_{k}}\right)\right) K\right] & =\angle\left(d_{k}^{*}\left(\frac{\mathrm{E}\left[z_{k}\right]}{h_{k}}\right)\right) K \\
& =\frac{\pi k(\beta-1)}{K}\left(K-1+2 N_{g}\right)
\end{aligned}
$$

therefore

$$
\begin{aligned}
\mathrm{E}\left[\hat{\beta}_{k}\right] & =\frac{\angle\left(d_{k}^{*}\left(\frac{\mathrm{E}\left[z_{k}\right]}{h_{k}}\right)\right) K}{\left(K-1+2 N_{g}\right) \pi k}+1 \\
& =\beta
\end{aligned}
$$

and clearly the expected value of (34) is $\beta$. 


\section{Cramer-Rao Lower Bound}

We follow the methodology given in [9] to derive the Cramer-Rao Lower bound (CRLB) for the joint estimation of $\beta$ and $\mathbf{b}$ using equation (14). We define the vector of unknowns as $\theta=\left[\bar{b}_{0}, \ldots, \bar{b}_{L-1}, \tilde{b}_{0}, \ldots, \tilde{b}_{L-1}, \beta\right]^{T}$, where $\bar{b}$ and $\tilde{b}$ are the real and imaginary parts of the channel impulse. From (14), we can see that $\tilde{\mathbf{y}}$ is a vector of random variables with distribution $N\left(\mathbf{W}_{\beta}^{H} \mathbf{D V} \mathbf{b}, \sigma_{q}^{2} \mathbf{I}\right)$. Therefore, the joint probability of $\tilde{\mathbf{y}}$ and $\theta$ is:

$p(\mathbf{y} \mid \theta)=\frac{1}{\left(\pi \sigma_{q}^{2}\right)^{K}} \exp \left\{-\frac{1}{\sigma_{q}^{2}}\left(\tilde{\mathbf{y}}-\mathbf{W}_{\beta}^{H} \mathbf{D V b}\right)^{H}\left(\tilde{\mathbf{y}}-\mathbf{W}_{\beta}^{H} \mathbf{D V b}\right)\right\}$

Since the covariance of the random samples is not dependent on the $\theta$, we can derive the $(r, c)$ element of the Fisher information matrix as:

$$
\begin{gathered}
{[\mathbf{I}(\theta)]_{r, c}=\frac{2}{\sigma_{n}^{2}} \operatorname{Re}\left\{\left[\frac{\partial \mu(\theta)}{\partial \theta_{r}}\right]^{H}\left[\frac{\partial \mu(\theta)}{\partial \theta_{c}}\right]\right\}} \\
\mu(\theta)=\mathbf{W}_{\beta}^{H} \mathbf{D V} \mathbf{b}
\end{gathered}
$$

Substituting (37) into (36), the Fisher Information matrix is:

$\mathbf{F I}=\frac{2}{\sigma_{q}^{2}}\left[\begin{array}{ccc}\operatorname{Re}\left\{\mathbf{F}^{H} \mathbf{F}\right\} & -\operatorname{Im}\left\{\mathbf{F}^{H} \mathbf{F}\right\} & -\operatorname{Im}\left\{\mathbf{F}^{H} \tilde{\mathbf{F}} \mathbf{b}\right\} \\ \operatorname{Im}\left\{\mathbf{F}^{H} \mathbf{F}\right\} & \operatorname{Re}\left\{\mathbf{F}^{H} \mathbf{F}\right\} & \operatorname{Re}\left\{\mathbf{F}^{H} \tilde{\mathbf{F}} \mathbf{b}\right\} \\ \operatorname{Im}\left\{(\tilde{\mathbf{F}} \mathbf{b})^{H} \mathbf{F}\right\} & \operatorname{Re}\left\{(\tilde{\mathbf{F}} \mathbf{b})^{H} \mathbf{F}\right\} & \operatorname{Re}\left\{(\tilde{\mathbf{F}} \mathbf{b})^{H} \tilde{\mathbf{F}} \mathbf{b}\right\}\end{array}\right]$

where we define $\mathbf{F}=\mathbf{W}_{\beta}^{H} \mathbf{D V}$ and $\tilde{\mathbf{F}}=\frac{j 2 \pi}{K} \mathbf{N}_{D} \mathbf{W}_{\beta}^{H} \mathbf{K}_{D} \mathbf{D V b}$ with $\mathbf{K}_{D}=$ $\operatorname{diag}[-K / 2, \ldots, 0, \ldots, K / 2-1]$ and $\mathbf{N}_{D}=\operatorname{diag}\left[N_{g}, \ldots, N_{g}+\right.$ $n-1]$.

The CRLB for the $i$ th element of $\theta$ is given by the $(i, i)$ element of $\mathbf{F I}^{-1}$. Note that (38) shows that the CRLB is dependent upon the specific channel realization $\mathbf{b}$. The modified CRLB (MCRLB) is formed by averaging the CRLB over the channel realizations.

In figure (5) we compare through simulation the performance of the MML and Ad-hoc Doppler rate estimators with the MCRLB. In our derivation for both estimators of $\beta$, we have assumed that we are able to perfectly estimate and compensate for $\epsilon$. However, it is expected that in practice there will be some residual carrier frequency offset due to imperfect estimation of $\epsilon$. Speth [10] gives an approximation to the additional noise (in the form of ICI) caused by a residual CFO of $\Delta \epsilon$ as $\sigma_{\Omega}^{2} \approx \frac{\pi^{2}}{3}(\Delta \epsilon T K)^{2}$. We show the performance of the two estimators in the presence of residual CFO, with the performance given as a function of SINR. The curves in figure (5) show that the non-Gaussian nature of the CFO error imposes a limit on the performance of the MML estimator at high SINR. Furthermore, we can see that at low SINR the MML estimator does not meet the MCRLB, which we expect from the approximation in (30). Finally, we can see that the MML estimator outperforms the Ad-hoc estimator.
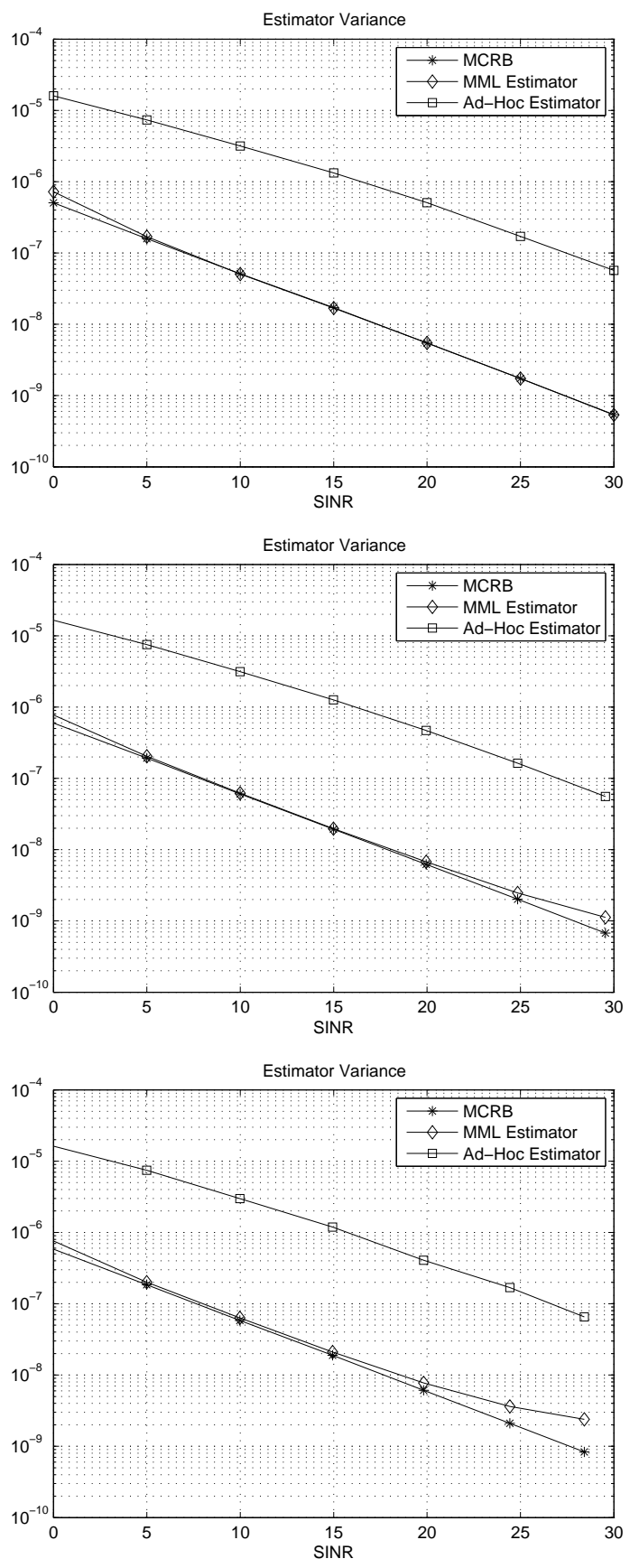

Fig. 5. Performance of the Ad-Hoc and MML Doppler Rate estimators compared to the Modified CRLB. In the top figure, the residual CFO is 0 and in the middle and bottom figures it is .5\% and $1 \%$ of the subcarrier spacing, respectively. The variance is plotted vs SINR, which includes the noise due to both AWGN and residual CFO 


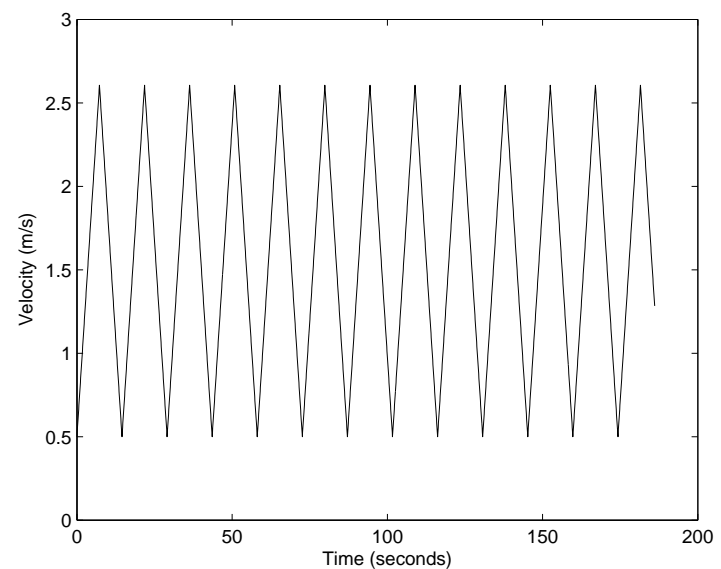

Fig. 6. Receiver Velocity

\section{Simulation Setup}

We simulate the performance of three receiver algorithms for OFDM with a changing Doppler rate. In the first receiver algorithm, we perform initial estimation of the Doppler rate via a pre-amble and then assume that the Doppler rate is constant over some duration, at which point we re-transmit the preamble to estimate a new Doppler rate. The second and third receiver designs track the Doppler rate, using the marginal maximum likelihood and Ad-hoc algorithms described in the preceding sections. We will denote these three approaches as init, MML, and Ad-hoc.

Our simulation set-up is as follows. Received packets are generated according to (13). The Doppler rate during packet $i$ is determined by the velocity of the source/receiver during packet $i$ by the equation $s_{i}=v_{i} / c$ where $c=1500 \mathrm{~m} / \mathrm{s}$ is the speed of sound in seawater. Figure (6) shows the velocity profile that we use in the simulation. The velocity ranges from $0.5 \mathrm{~m} / \mathrm{s}$ to $2.6 \mathrm{~m} / \mathrm{s}$. We assume that the craft accelerates and decelerates at approximately $0.29 \mathrm{~m} / \mathrm{s}^{2}$.

In our simulation, the channel coefficients and the Doppler rate are constant over an OFDM symbol. The Doppler rate changes from symbol to symbol as described in the previous paragraph. We simulate a channel with 10 taps with an exponentially decaying power delay profile, the power delay profile is shown in figure (7). Without loss of generality, the tap weights are chosen so that $E\left[\left|H\left(f_{k}\right)\right|^{2}\right]=\sum_{l=0}^{L-1} E\left[\left|b_{l}\right|^{2}\right]=1$. The channel taps are modeled as iid and individually Rayleigh fading on each tap.

We transmit OFDM symbols with $\mathrm{K}=256$ subcarriers and $\mathrm{Ng}=10$ guard symbols. The data symbols are sub-modulated using 4-PSK modulation, and every fourth subcarrier contains a probe symbol. The frequency band that we use is $10-14$ $\mathrm{KHz}$, and we use a raised cosine pulse shaping filter with a rolloff factor of 0.4 . Therefore, the $T_{s}$ is approximately $93 \mathrm{~ms}$, and the velocity changes by $+/-.027 \mathrm{~m} / \mathrm{s}$ over each OFDM symbol with a corresponding change in the Doppler rate of $1.8 * 10^{-} 5$.

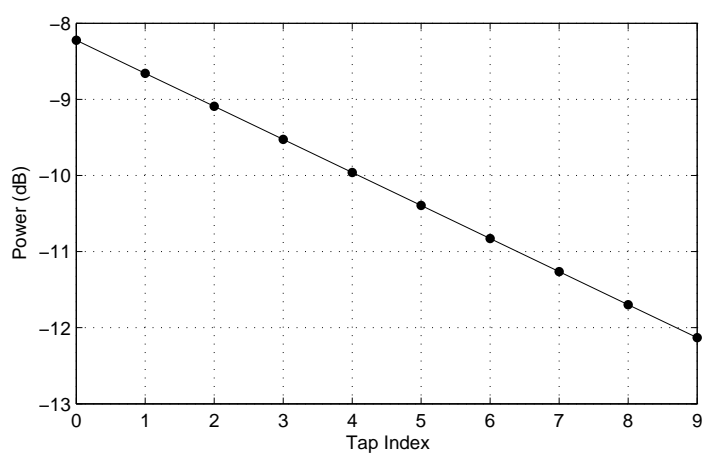

Fig. 7. Power Delay Profile

For each receiver, we assume that the initial Doppler rate is estimated perfectly during the pre-amble. The performance of each receiver is given as a function of the SNR, which we define as:

$$
\mathrm{SNR}=\frac{\mathrm{E}\left[\left|d_{k} H\left(f_{k}\right)\right|^{2}\right]}{\sigma_{q}^{2}}
$$

We show the results in figures (8), (9), and (10). Figure (8) shows that the variance of both the MML estimator and Ad-Hoc estimator exceeds the MCRLB; however, since we are not accounting for the additional noise imposed by the residual $\mathrm{CFO}$, this is to be expected. It is also noted that at $0 \mathrm{~dB}$, the performance of the MML estimator is closest to the MCRLB, which is not expected. This is most likely due to the resolution of the MML Doppler Rate estimator (ie, the range of $\beta$ over which the estimator is searching). This figure also shows that we do not see the lower bound on the MML estimate due to CFO error which was observed in figure (5). The explanation for this is given in figure (9) where it can be seen that as the SNR improves, so does the CFO estimate. The net effect of this is that MML Doppler Rate estimate continuously improves with SNR.

Finally, we show the uncoded symbol error rate in figure (10). At low SNR, the Ad-hoc estimator performs very poorly because it makes estimates of the Doppler rate that deviate further from the true Doppler rate than does the initial estimate. This causes an increase in ICI, and thus poor performance. However, at high SNR both the of the receivers with Doppler rate tracking perform better than that using only an initial estimate of the Doppler rate. Of course, the performance of the 'initial only' estimator could be improved by re-estimating the Doppler rate more often, but this would also incur increased overhead and a lower data rate.

\section{CONCLUSION}

We have described a model for OFDM symbol reception in an underwater channel where the Doppler rate varies from symbol to symbol, and we have proposed two methods for tracking this changing Doppler rate. The receiver design that we have proposed builds upon previous works which estimated the Doppler rate via a pre/post-amble and assumed that it was 


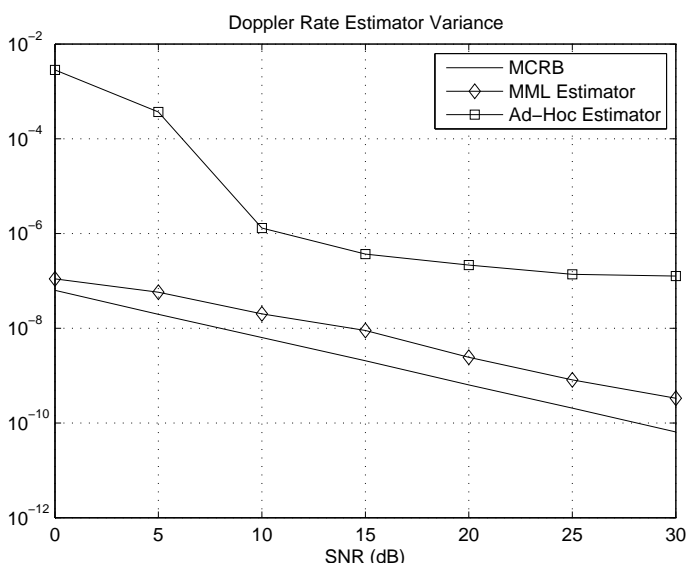

Fig. 8. Doppler Rate Estimator Variance

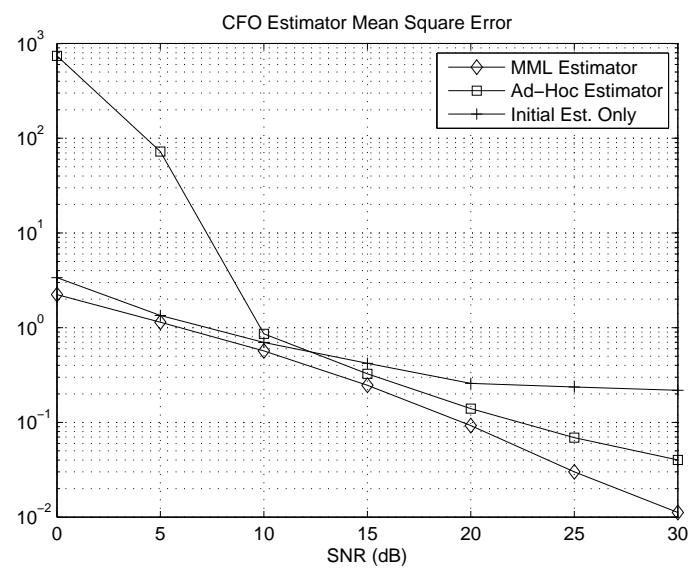

Fig. 9. Carrier Frequency Offset estimator MSE

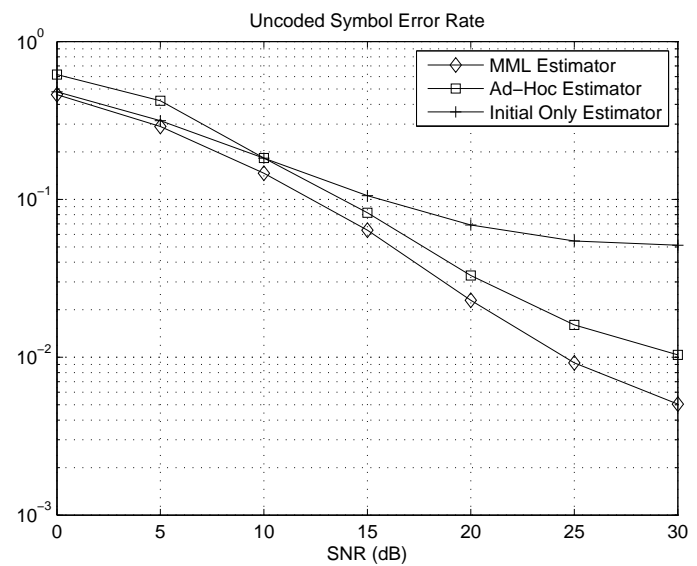

Fig. 10. Uncoded Symbol Error Rate constant over a block duration. Of the two Doppler rate estimators that we have proposed, the MML estimator shows better performance, but is more complex. Future work will invovle testing the proposed receiver design via experimentation in underwater channels.

\section{ACKNOWLEDGMENT}

This work was supported by the NASA Earth Science Technology Offices Advanced Information Systems Technology (AIST) Program under award number AIST-05-0030.

\section{REFERENCES}

[1] R. Stokey, A. Roup, C. von Alt, B. Allen, N. Forrester, T. Austin, R. Goldsborough, M. Purcell, F. Jaffre, G. Packard, and A. Kukulya, "Development of the remus 600 autonomous underwater vehicle," Sept. 2005, pp. 1301-1304 Vol. 2.

[2] B. Li, S. Zhou, M. Stojanovic, L. Freitag, and P. Willett, "Nonuniform doppler compensation for zero-padded ofdm over fast-varying underwater acoustic channels," June 2007, pp. 1-6.

[3] S. Mason, C. Berger, S. Zhou, and P. Willett, "Detection, synchronization, and doppler scale estimation with multicarrier waveforms in underwater acoustic communication," April 2008, pp. 1-6.

[4] B. Sharif, J. Neasham, O. Hinton, and A. Adams, "A computationally efficient doppler compensation system for underwater acoustic communications," Oceanic Engineering, IEEE Journal of, vol. 25, no. 1, pp. 52-61, Jan 2000.

[5] B. Li, S. Zhou, M. Stojanovic, and L. Freitag, "Pilot-tone based zp-ofdm demodulation for an underwater acoustic channel," Sept. 2006, pp. 1-5.

[6] G. Giunta, "Fast estimators of time delay and doppler stretch based on discrete-time methods," Signal Processing, IEEE Transactions on, vol. 46, no. 7, pp. 1785-1797, Jul 1998.

[7] U. Mengali and A. N. D'Andrea, Synchronization Techniques for Digital Receivers. New York: Plenum Press, 1997.

[8] M. Stojanovic, "Low complexity ofdm detector for underwater acoustic channels," Sept. 2006, pp. 1-6.

[9] S. M. Kay, Fundamentals of Statistical Signal Processing: Estimation Theory. Englewood Cliffs, NJ: Prentice Hall, 1993.

[10] M. Speth, S. Fechtel, G. Fock, and H. Meyr, "Optimum receiver design for wireless broad-band systems using ofdm. i," Communications, IEEE Transactions on, vol. 47, no. 11, pp. 1668-1677, Nov 1999. 